\title{
Novel Fluid Dynamics Model to Predict Draw of Hollow Core Photonic Band-Gap Fibres
}

\author{
Gregory T. Jasion ${ }^{(1)}$, Seyed Reza Sandoghchi ${ }^{(1)}$, Yong Chen ${ }^{(1)}$, Natalie V. Wheeler ${ }^{(1)}$, Tom Bradley ${ }^{(1)}$, \\ Naveen Baddela ${ }^{(1)}$, John Hayes ${ }^{(1)}$, Marco Petrovich ${ }^{(1)}$, David J. Richardson ${ }^{(1)}$, John S. Shrimpton ${ }^{(2)}$ and \\ Francesco Poletti ${ }^{(1)}$ \\ (1) Optoelectronics Research Centre, University of Southampton, Southampton, SO17 1BJ, United \\ Kingdom, g.jasion@soton.ac.uk \\ (2) Aerodynamics and Fluid Mechanics, Faculty of Engineering and the Environment, University of \\ Southampton
}

\begin{abstract}
A$ method to track the evolution of a microstructured fibre, from initial preform to final fibre geometry, is presented. Up scaling to longer lengths, new structure development and effects of material parameters can all be explored with this model.
\end{abstract}

\section{Introduction}

Hollow Core Photonic Band Gap Fibres (HCPBGFs) are a rapidly developing section of fibre research. The structure must be delicately controlled to provide the correct band-gap and efforts to reduce loss demand increasing precision of node position and strut thickness. Practically, these fibres are drawn and designed using experience and trial and error; a process that is both expensive and time consuming. When specific and unconventional structures need to be targeted then a predictive tool would rapidly speed up the development cycle and reduce the number of iterations required.

Current simulation methods, applying conventional computational fluid dynamics approaches of microstructure fibres, are in development but still limited to a small number of holes ${ }^{1,2}$. The limitations are intrinsic in the nature of the method, finite volume or difference methods require a high mesh density to resolve boundaries effectively and high aspect ratio volumes need to be avoided. Even for a 2D holey fibre the mesh size required is high. For an HC-PBGF where the struts are thin, with an aspect ratio $>20$, the mesh density required for the full structure is prohibitively high.

A computational method has many applications such as considering how different materials might behave, novel structures evolve and industrial length up-scaling. As these simulations are relatively cheap, one can search the design space by casting a wide net of parameter combinations. The migration from research scale fabrication to industrial scale fabrication requires preforms to become significantly larger, draw speeds of the order of $\mathrm{km} / \mathrm{min}$ and consequently hotter furnaces. This model can help understand the changes in draw parameters required for volume up-scaling. The kernel of the method currently sets up hexagonal lattice geometries but could easily be adapted to solve any lattice based microstructure, such as anti-resonance or suspended-core microstructure fibres.

In this paper we present a simple and flexible method to model the structure of a hollow core microstructured fibre. First, the method structure and governing equations are outlined; we follow this with a simple set of results and compare them with Scanning Electron Micrographs (SEMs) of real HCPBGFs. Finally we comment on the capabilities of this model.

\section{Method}

To simulate the evolution of the microstructure during the draw we split the simulation process into two parts: the external jacket glass is modelled like a simple drawn capillary using the model of Fitt et al. ${ }^{3}$. The Fitt model predicts the inner and outer diameters of a drawn capillary for a non-isothermal draw with applied pressure and surface tension. The internal microstructure, Fig. 1, uses that capillary as a boundary while solving physical equations that govern its motion. Fig. 2 shows the neck down generated using the Fitt model. The simulation starts with a 2D preform geometry, Fig. 1, and marches in the $z$ direction-the direction of fibre draw. The

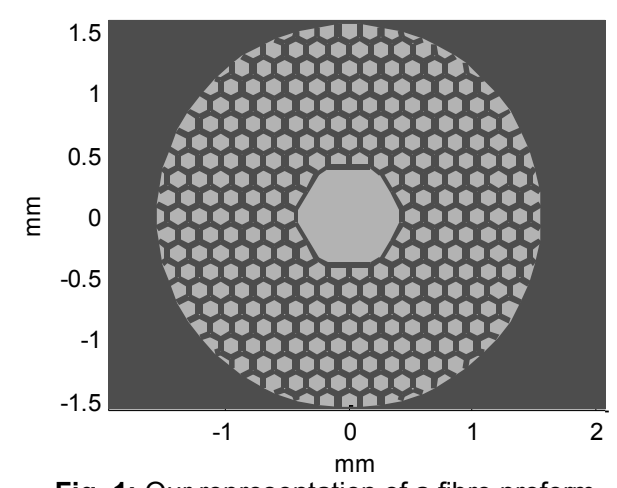

Fig. 1: Our representation of a fibre preform microstructure, the jacket glass is $3 \mathrm{~mm}$ thick. 


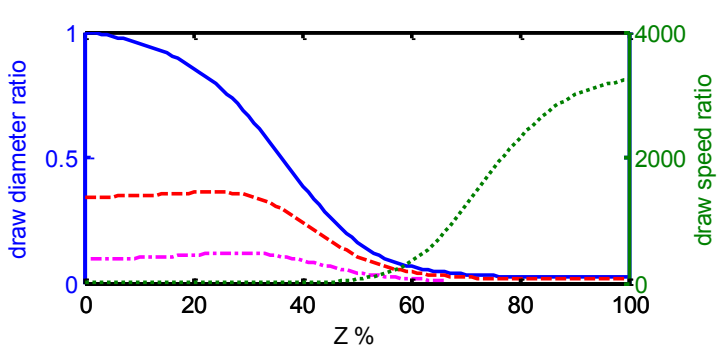

Fig. 2: Draw profile produced by the Fitt model. external OD (-), internal OD (-- -), core (-+-+-), speed $(+*)$.

Fitt model governs the boundary motion, while the forces of pressure, viscosity and surface tension are applied to the microstructure which evolves accordingly.

Consider the most typical HC-PBGF preform; it is a triangular lattice of capillaries. We replace this with a honeycomb like structure of rectangular struts within a boundary defined by the jacket glass, Fig. 1 shows our representation of the structure. Each strut connects 2 nodes, and each node is a junction of 3 struts. We now consider each of these struts to be a 1D control volume of fluid upon which we apply forces of viscosity due to strut extension rate (Eq. 1), gas pressure (Eq. 2) and surface tension from curvature at the junction between struts (Eq. 3), Fig. 3.

$$
F_{v}=2 \mu \frac{d \vec{u}}{d \vec{s}} w D \hat{s}
$$

where $\mu$ is the glass viscosity, $\frac{d \vec{u}}{d \vec{s}}$ is the rate of change of extension in the direction of the strut, $w, D$ and $\hat{s}$ are the width, depth and unit vector along the length of the strut.

$$
F_{p}=\left(P_{A}-P_{B}\right) D l \hat{n}
$$

where $P_{A}-P_{B}$ is the pressure difference on either side of the strut, $l$ and $\hat{n}$ are the length and normal unit vector of the strut.

$$
F_{s}=\frac{\gamma}{R_{C}} A_{c} \vec{c}
$$

where $\gamma$ is the material surface tension value, $R_{c}$ is the radius of curvature at the junction between struts, $A_{c}$ is the projected area of the curved region and $\vec{c}$ is the direction the force acts. The curvature can be obtained either with a fitted arc or spline. The viscosity, $\mu$, is a function of the temperature ${ }^{4}$. The furnace profile was measured from our furnace. We assume the glass temperature is a function of $z$ only.

Self-pressurised draws can be simulated by using a gas volume conservation relationship to change cell pressure, $p_{1}$, as a function of gas volume on a cell by cell basis by comparing the new volume, $V_{1}$, with the original volume, $V_{0}$, and

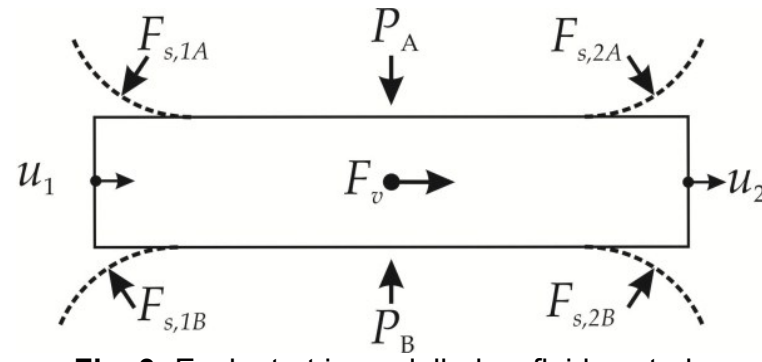

Fig. 3: Each strut is modelled as fluid control volume, with forces of pressure, viscosity and surface tension.

ambient pressure, $p_{0}$, using $p_{0} V_{0}=p_{1} V_{1}$, no other pressure is applied. The mass of each strut is conserved, i.e. if struts are stretched they become thinner.

The forces on each of the struts are resolved to the node and a numerical scheme finds the velocities and positions in time and $z$. The simulation finishes when the slice exits the furnace.

This computational model gives us access to the in-furnace dynamics - we can see the evolution of the microstructure in the hot zone. The dynamics in the furnace are governed by a delicate balance between the forces of surface tension and pressure and viscosity. In this manner the model can help us understand the physical effects occurring throughout the furnace rather than just the final fibre shape.

\section{Preform Structure}

We performed a simulated draw of a 19-cell HCPBGF with 7 cladding rings ${ }^{5}$ and compare the final fibre structures with SEMs of real fibres drawn from a similar preform. The simulated cane has a lattice of holes with a 100 micron radius, and 50 micron strut thickness with a $3 \mathrm{~mm}$ jacket tube, Fig. 1.

\section{Results}

Here we demonstrate that this model can simulate fibre draws from preform to fibre and produce realistic fibre structures based on physical forces.

The simulated preform was drawn to fibres with OD of 200 micron and a range of expansions (internal/external diameter of jacket) using the Fitt model.

Fig. 4 shows the simulated fibre microstructures for 3 expansion ratios of $50 \%$, $60 \%$ and $70 \%$. It is clearly evident that the jacket glass and struts become thinner with higher expansion ratios as we would expect. The cells surrounding the core become increasingly elliptical and the corner holes show increasing node spacing as the fibre is expanded.

Compare these simulated structures with the SEMs of similar fibres shown in Fig. 5, these fibres are expanded to $58 \%, 65 \%$ and $74 \%$. The 

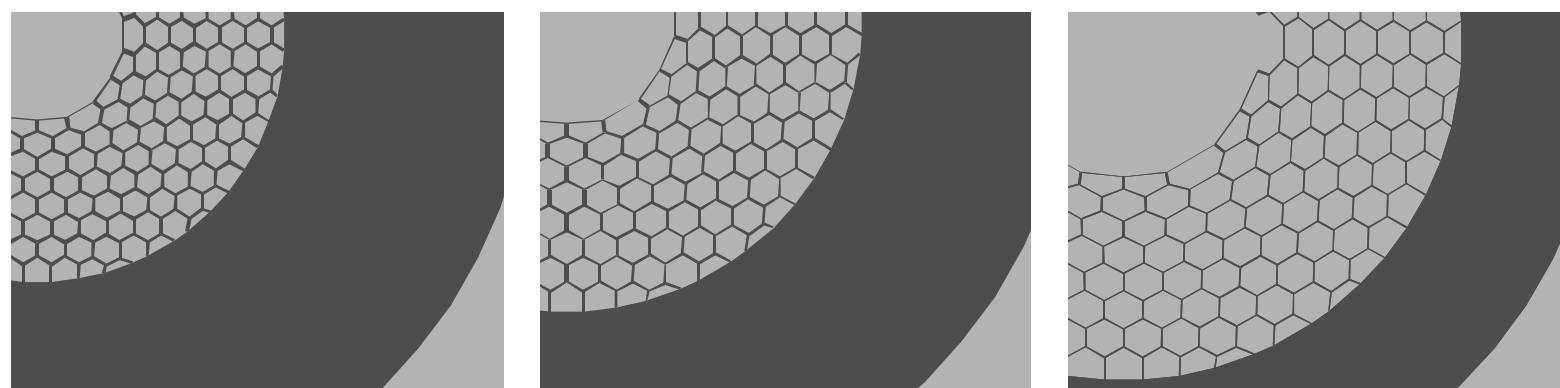

Fig. 4: Results of simulating draw of 19-cell 7-row HC-PBGF drawn to different expansions from the same preform, Fig. 1. Fibre expansion ratio (internal diameter/ outer diameter of jacket glass), from the left:
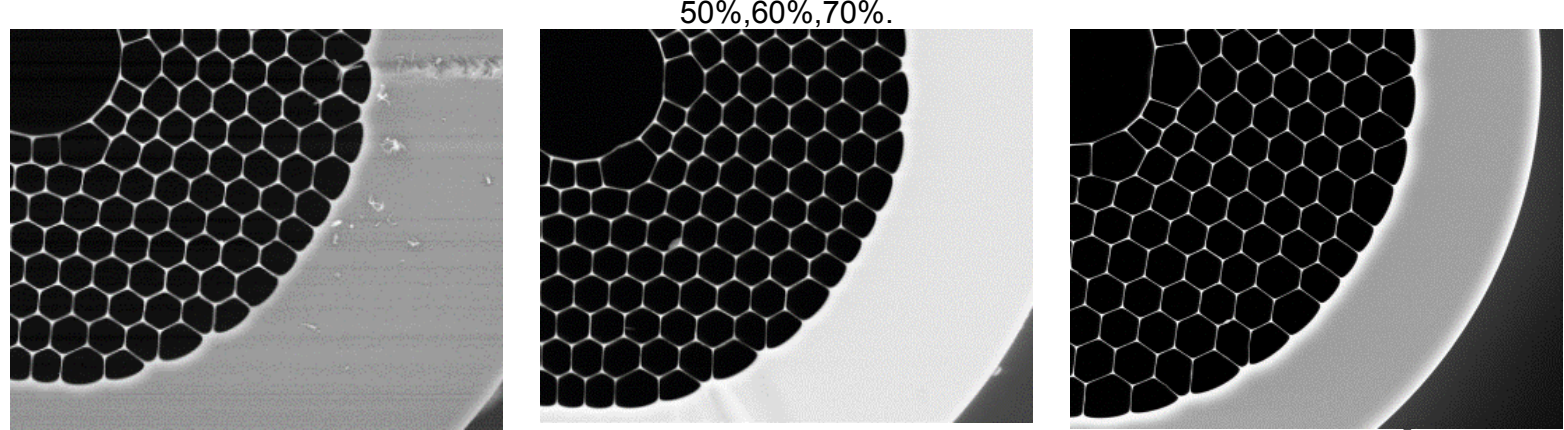

Fig. 5: SEM images of 19-cell 7-row fibres drawn to different expansions from the same preform ${ }^{5}$. Fibre expansion ratio (internal diameter/ outer diameter of jacket glass), from the left: $58 \%, 65 \%, 74 \%$.

honeycomb-lattice is regular and balanced in both SEM and simulations. The distance between the corner nodes in the core increases as the fibre expands, this feature is accurately portrayed by the simulation.

The core of the fibres seen in the SEMs is less expanded than the predicted by the simulations. This difference can be attributed to the fact that pressure system used in the simulation is a simplification of the pressure control system used during the fabrication process. The SEMs show the slight deviation of the first cladding ring surrounding the hollow core in relation to the uniform honeycomb cladding structure. The pressurisation of the core and cladding cells is being investigated to better understand this phenomenon.

\section{Capability}

The model presented here is a powerful tool for accurately predicting the microstructure of a $\mathrm{HC}$ PBGF drawn from a cane with a hexagonal lattice structure which has been used to draw low loss HC-PBGFs ${ }^{6}$. The model offers the potential scope for rapid optimisation of fibre fabrication parameters by predicting the necessary drawing conditions a priori. This potential can then be harnessed to up-scale the lengths of HC-PBGF which can feasibly be drawn from a single preform therefore maximising the potential yield. Combining this model with a finite element model that can predict the optical properties of the fibre allows for the virtual draw and characterisation of fibres. The proposed system is then tasked to rapidly search and optimise preform designs and draw parameters to produce low loss fibres for a specified wavelength.

\section{Acknowledgements}

This work was supported by the Royal Society and by the EU FP7-ICT under grant 258033 (MODE-GAP).

\section{References}

[1] Luzi et al., "Numerical Solution and Experimental Validation of the Drawing Process of Six-Hole Optical Fibers Including the Effects of Inner Pressure and Surface Tension," J. Lightw. Technol., Vol. 30, no. 9, p. 1306 (2012).

[2] S. C. Xue et al., "Fabrication of microstructured optical fibers-Part II: Numerical modeling of steady-state draw process," J. Lightw. Technol., Vol. 23, no. 7, p. 2255 (2005).

[3] A. D. Fitt et al., "The mathematical modelling of capillary drawing for holey fibre manufacture," J. Eng. Math., Vol. 43, p. 201 (2002).

[4] G. Urbain et al., "Viscosity of liquid silica, silicates and alumino-silicates," Geochimica et Cosmochimica Acta, Vol. 46, no. 6, p. 1061 (1982).

[5] Y. Chen et al., "Understanding wavelength scaling in 19cell core hollow-core photonic bandgap fibers," Proc. OFC, M2F.4, San Francisco (2014).

[6] N.K. Baddela et al., "First demonstration of a low loss 37-cell hollow core photonic bandgap fiber and its use for data transmission," Proc. CLEO, CTU2K.3, San Jose (2013) 